\title{
Broadband subwavelength grating mirror and its application to vertical-cavity surface- emitting laser
}

Chung, II-Sug; Mørk, Jesper; Gilet, Philippe; Chelnokov, Alexei

Published in:

Transparent Optical Networks, 2008.

Link to article, DOI:

10.1109/ICTON.2008.4598602

Publication date:

2008

Document Version

Publisher's PDF, also known as Version of record

Link back to DTU Orbit

Citation (APA):

Chung, I-S., Mørk, J., Gilet, P., \& Chelnokov, A. (2008). Broadband subwavelength grating mirror and its application to vertical-cavity surface-emitting laser. In Transparent Optical Networks, 2008.: ICTON 2008. 10th Anniversary International Conference on (pp. Tu.C2.5). IEEE. https://doi.org/10.1109/ICTON.2008.4598602

\section{General rights}

Copyright and moral rights for the publications made accessible in the public portal are retained by the authors and/or other copyright owners and it is a condition of accessing publications that users recognise and abide by the legal requirements associated with these rights.

- Users may download and print one copy of any publication from the public portal for the purpose of private study or research.

- You may not further distribute the material or use it for any profit-making activity or commercial gain

- You may freely distribute the URL identifying the publication in the public portal 


\title{
Broadband Subwavelength Grating Mirror and Its Application to Vertical-Cavity Surface-Emitting Laser
}

\author{
Il-Sug Chung ${ }^{1}$, Jesper Mørk ${ }^{1}$, Philippe Gilet ${ }^{2}$, Alexei Chelnokov ${ }^{2}$ \\ ${ }^{I}$ Technical University of Denmark, DK-2800 Kgs. Lyngby, Denmark \\ ${ }^{2}$ CEA-G Léti MINATEC, Grenoble, France \\ Tel: (45) 4525 6383,Fax: (45) 45256581,e-mail: isc@com.dtu.dk
}

\begin{abstract}
Various high-index-contrast sub-wavelength grating (HCG) mirror designs have been investigated. It reveals that transverse magnetic (TM-) and transverse electric (TE-) HCG reflect the incident fields in quite different ways and that the TM-HCG enables very thin gap below the grating. Based on these results, a new HCG VCSEL design with a thin oxide gap has been suggested. The thin oxide gap structure has a number of advantages including easier fabrication, better mechanical stability, and very strong single-mode properties.
\end{abstract}

Keywords: grating, photonic crystal, VCSEL.

\section{INTRODUCTION}

Gratings have been an old friend of photonics engineers as couplers, polarizers, spectrometers, etc. Recentlyreported broadband reflection property of gratings attracts our attention again to our old friend. A single layer subwavelength grating surrounded by low index medium can be highly reflective to a specific polarization, and its reflective bandwidth can be much broader than that of conventional distributed Bragg reflectors (DBRs). Since their typical index contrast is high, we call them high-index-contrast subwavelength grating (HCG) in this paper.

Using a HCG in VCSELs as a top mirror can bring many novel advantages. (1) Very strong polarization stability can be obtained for reducing noise due to polarization switching. (2) Very strong single mode property can be achieved, which potentially enables HCG VCSELs to have high single-mode output power. (3) Waferbonding of a GaAs/AlGaAs top mirror in the fabrication of InP-based long wavelength VCSELs can be avoided. (4) Light mass of the HCG enables an efficient tunable HCG VCSEL with 30-times faster tuning speed and 1/1000-times smaller power consumption than the conventional DBR-based tunable VCSEL.

To further optimize HCG VCSELs, it is essential to understand the characteristics of HCG itself. However, no comparison of various HCG designs in a common standard has been done so far. In this paper, the mirror characteristics of various HCG designs are numerically investigated. Then, based on these result, a new HCG structure with a thin oxide gap is suggested. Finally, single-mode property of HCG VCSEL is discussed in comparison with the state-of-the-art high-power single-mode VCSEL, i.e., surface-relief VCSEL.

\section{HCG MIRROR}

Various HCG designs have been investigated by using finite-difference time-domain (FDTD) method. The parameters of the investigated HCG designs are summarized in Table 1. The HCGs can be categorized into TMand TE-HCGs, depending on the reflective polarization. TM-(TE-) HCG has very high reflectivity selectively for an incident electric field perpendicular (parallel) to the grating direction. The main task of this section is to find out the difference between TE- and TM-HCGs.

Table 1. Summary of the investigated HCG designs

\begin{tabular}{l|cccccc}
\hline Sample name & $\begin{array}{c}\text { Reflective } \\
\text { polarization }\end{array}$ & $\begin{array}{c}\text { Wavelength } \\
\lambda\end{array}$ & $\begin{array}{c}\text { Period } a_{g} \\
\left(a_{\mathrm{g}} / \lambda\right)\end{array}$ & $\begin{array}{c}\text { Thickness } h_{g} \\
\left(n_{\mathrm{g}} h_{g} / \lambda\right)\end{array}$ & $\begin{array}{c}\text { Filling ratio } \\
\alpha\end{array}$ & $\begin{array}{c}\text { Refractive } \\
\text { index, } n_{\mathrm{g}} / n_{\text {air }}\end{array}$ \\
\hline TE1 [1] & TE & $1550 \mathrm{~nm}$ & $1150 \mathrm{~nm}(0.74)$ & $255 \mathrm{~nm}(0.52)$ & $35 \%$ & $3.17 / 1$ \\
TE2 [2] & TE & $1550 \mathrm{~nm}$ & $1200 \mathrm{~nm}(0.77)$ & $670 \mathrm{~nm}(1.53)$ & $50 \%$ & $3.53 / 1$ \\
TM1 [3] & TM & $850 \mathrm{~nm}$ & $420 \mathrm{~nm}(0.49)$ & $161 \mathrm{~nm}(0.68)$ & $68 \%$ & $3.60 / 1$ \\
TM2 [4], [5] & TM & $980 \mathrm{~nm}$ & $391 \mathrm{~nm}(0.40)$ & $255 \mathrm{~nm}(0.92)$ & $62 \%$ & $3.53 / 1$ \\
\hline
\end{tabular}

\subsection{Computational settings}

Figure 2(a-TE2) and Figure 2(a-TM2) show computational domains. The investigated HCGs are suspended in the air. Domain boundary is $1 \lambda$ away from the HCG. The whole domain is surrounded by perfectly-absorbing layers (PML). The $3 \lambda$-wide source is incident from the bottom. Its amplitude is normalized to unity. A $5 \lambda$-wide detector is located just below the source.

\subsection{Results and discussion}

All the results are summarized in Table 2. Detailed analysis for TE2 and TM2 are given in Figure 1. The results of TE1 and TM1 designs are similar as those of TE2 and TM2 designs, respectively. 

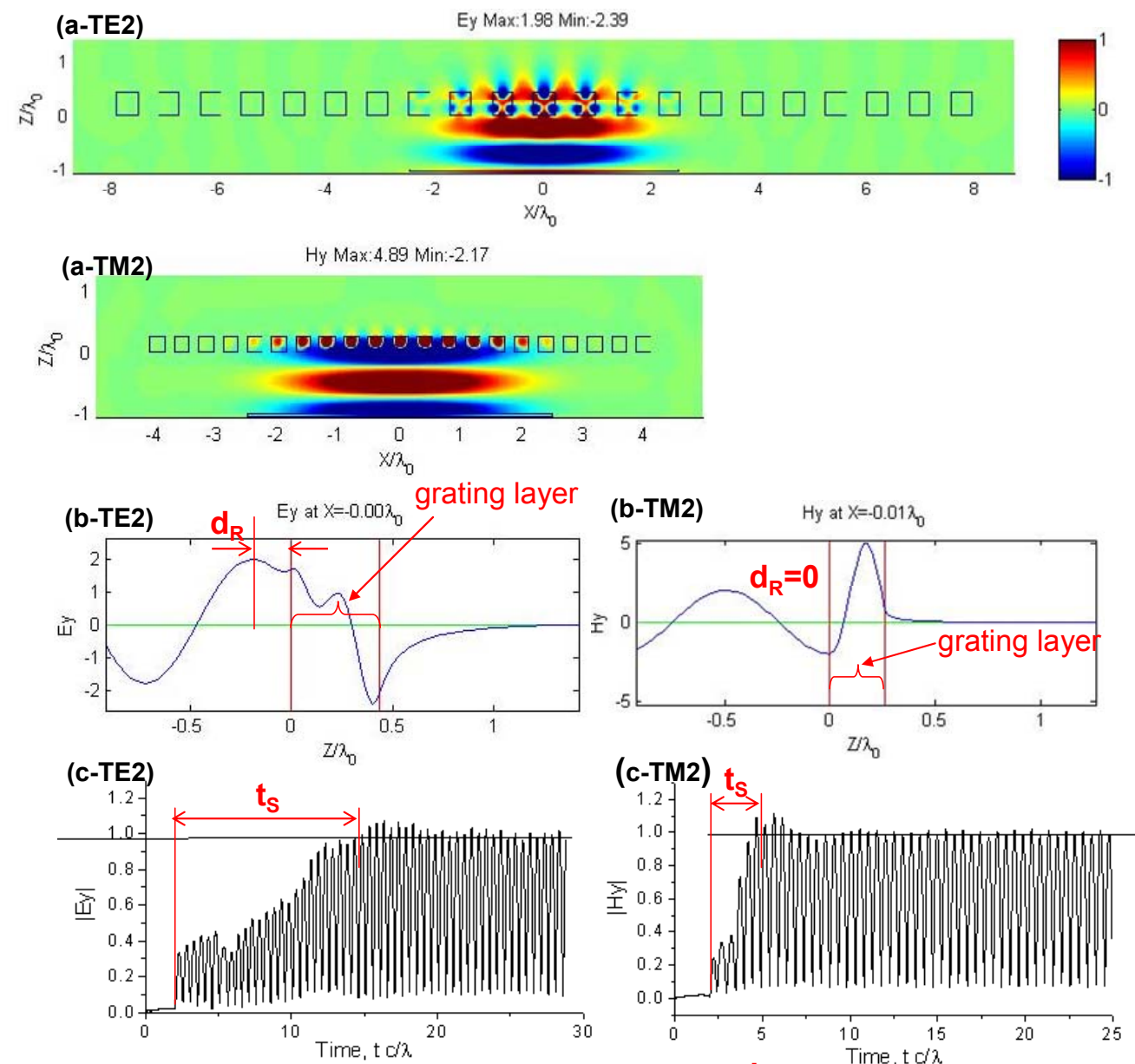

(d-TE2)

(d-TM2) .... $\mathrm{d}_{\mathrm{s}}$

At $-0.01 \lambda_{0}$ below the grating, $1 / \mathrm{e}^{2}$-width: $1.73 \lambda_{0}$

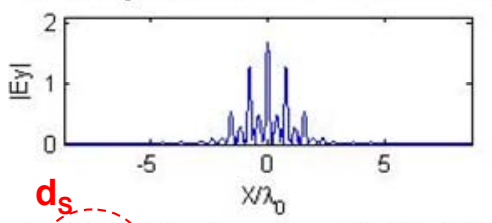

Át $-0.01 \lambda_{D^{\prime}}^{\prime}$ below the grating, $1 / \mathrm{e}^{2}$-width: $2.98 \lambda_{0}$

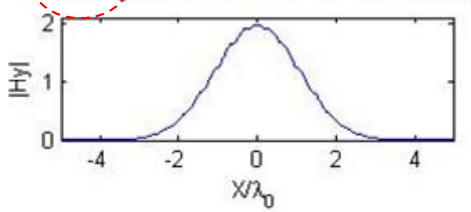

At $-0.70 \lambda_{0}$, below the grating, $1 / \mathrm{e}^{2}$-width: $2.99 \lambda_{0}$

At $-0.50 \lambda_{0}$ below the grating, $1 / \mathrm{e}^{2}$-width: $3.01 \lambda_{0}$
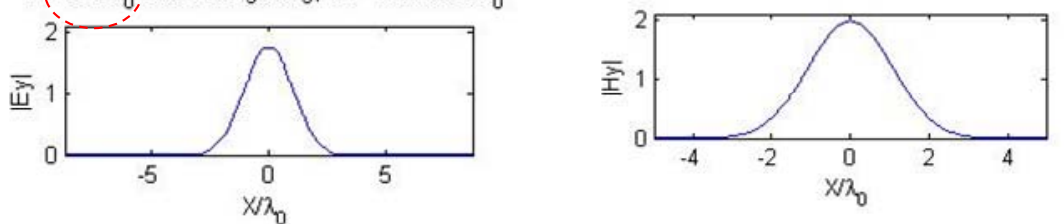

Figure 1. Simulation results of TE2 and TM2 designs.

Table 2. Summary of HCG simulations.

\begin{tabular}{c|cccc}
\hline Sample name & $\begin{array}{c}\text { Reflective bandwidth } \\
(R>99 \%)\end{array}$ & Phase shift, $d_{R}$ & Stabilization time, $t_{S}$ & Gap thickness, $d_{S}$ \\
\hline TE1 & $174 \mathrm{~nm}$ & $0.14 \lambda$ & $18 \mathrm{fs}$ & $>0.6 \lambda$ \\
TE2 & $33 \mathrm{~nm}$ & $0.20 \lambda$ & $66 \mathrm{fs}$ & $>0.6-0.7 \lambda$ \\
TM1 & $43 \mathrm{~nm}$ & $0.04 \lambda$ & $9.7 \mathrm{fs}$ & $\sim 0$ \\
TM2 & $186 \mathrm{~nm}$ & $0.01 \lambda$ & $8.8 \mathrm{fs}$ & $\sim 0$ \\
\hline
\end{tabular}

TM HCG gives smaller reflection phase shift. Figure 1(b-TE2) is the x-cut image of Figure 1(a-TE2) at $x=0$. The field in $(Z<0)$ region is the sum of the incident wave from the source and the reflected wave from the grating, while the field in $\left(Z>0.43 \lambda_{0}\right)$ region is only transmitted wave from the grating. The first field intensity maximum in $(Z<0)$ occurs at $Z=-d_{R}=-0.20 \lambda_{0}$. The amount of $d_{R}$ is related with a reflection phase shift due to 
resonance coupling inside the grating. This is important when a HCG is combined with a DBR. In the combination case, the optical thickness of the spacing between the bottom surface of the grating and the top surface of the DBR should be odd multiples of $\lambda_{0} / 4+d_{R}$ to keep the reflections from the grating and DBR in phase. The $d_{R}$ is almost zero for TM HCG, as shown in Figure 1(b-TM2) and Table 2. Thus, the spacing thickness between the DBR and grating should be just odd multiples of $\lambda_{0} / 4$.

Stabilization time, $t_{s}$ is defined as the time that is needed for the intensity of the reflected field to reach its steady-state value, as shown in Figure 1(b-TE2) or (b-TE2). In Table 2, TE-HCGs take considerably longer time to reach steady state than TM-HCG. It indirectly shows that the strength of resonance coupling in TM-HCG is much weaker than that in TE-HCG, since the coupling from the incident free space mode to the waveguide mode in the grating and the reverse coupling from the waveguide mode to free space mode takes some time. More detailed discussion will be given in the presentation.

In real devices, the HCG needs an air gap or low index material gap, as shown in Figure 2(b) and (c). How thick this gap needs to be? Gap thickness, $d_{s}$ is defined as a gap thickness that is needed the reflected wave to recover the Gaussian shape of the incident wave. In TE-HCGs, $0.6-0.7 \lambda_{0}$ is needed as shown in Figure $1(\mathrm{~d}$ TE2), whereas in TM-HCGs, no distance is necessary as shown in Figure 1(d-TM2).

In real HCG VCSELs, 4-5 pairs of DBR are combined with HCG to efficiently spread injected carriers. From the above discussions, an ideal gap thickness between the HCG and DBR, $h_{\text {gap }}$ should be

$$
h_{\text {gap }}=\frac{(2 m+1) \lambda_{0} / 4+d_{R}}{n_{\text {gap }}}, \text { where } m=0,1,2, \cdots
$$

and $n_{\text {gap }}$ is the refractive index of the gap. Specifically, the thinnest gap thicknesses for TE1-, TE2-, TM1-, and TM2-HCGs are $0.89 \lambda_{0} / n_{\text {gap }}, 0.95 \lambda_{0} / n_{\text {gap }}, 0.29 \lambda_{0} / n_{\text {gap }}$, and $0.26 \lambda_{0} / n_{\text {gap }}$, respectively.

\section{GRATING MIRROR VCSEL}

TM2 design is chosen for HCG VCSEL simulations to reduce the gap thickness as much as possible.

\subsection{Device structure}

(a)

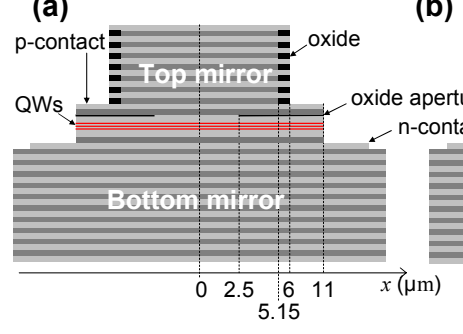

(b)

(b) air gap $h_{A G}=1226.3(\mathrm{~nm})$ (c) $h_{O G}=153.3(\mathrm{~nm})$

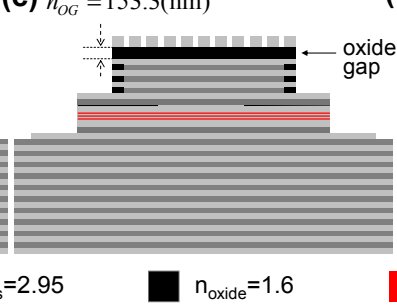

(d)

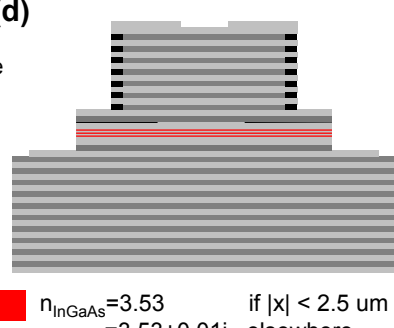

Figure 2. Schematic device structures of investigated (a) plain VCSEL, (b) HCG VCSEL with a thick air gap, (c) HCG VCSEL with a thin oxide gap, and (d) surface-relief VCSEL.

The investigated VCSEL structure is based on the $980 \mathrm{~nm}$ epi design with a thin oxide at anti-node position. More detailed description of device structure and simulation method can be found in [5]. The air gap in Figure 2 (b) is $5 \lambda_{0} / 4$ thick. However, the oxide gap in Figure 2(b) is $\lambda_{0} / 4$ thick which is the thinnest thickness from the result of previous section.

The extent of suppression of higher-order modes is quantified by the mode stability factor $S$, defined as:

$$
S=\frac{g_{1}-g_{0}}{g_{0}} \times 100(\%)
$$

where $g_{0}$ and $g_{1}$ represent the threshold gains of the fundamental and first-order modes, respectively.

\subsection{Result and discussion}

Thin oxide gap design using TM HCG has almost same properties as thick oxide gap design. They have similar well-defined optical modes, as shown in Figure 1(a) and (b). Their threshold gain and mode stability factor are also almost same, as shown in

Figure 3.

The single mode property of HCG VCSEL can be greatly improved by adjusting the HCG region size. The higher order-modes can be selectively suppressed by reducing the HCG region, as shown in

Figure 3(c). The optimal HCG size is the oxide aperture size (13 periods in the considered design). The single-mode property of the optimized HCG VCSEL is stronger than that of the state-of-the-art surface relief VCSEL, as shown in Figure 4. It is because the spatial reflectivity contrast of the HCG VCSEL is much larger (a few tens of percents) than that of the surface relief VCSEL (a few percents). 


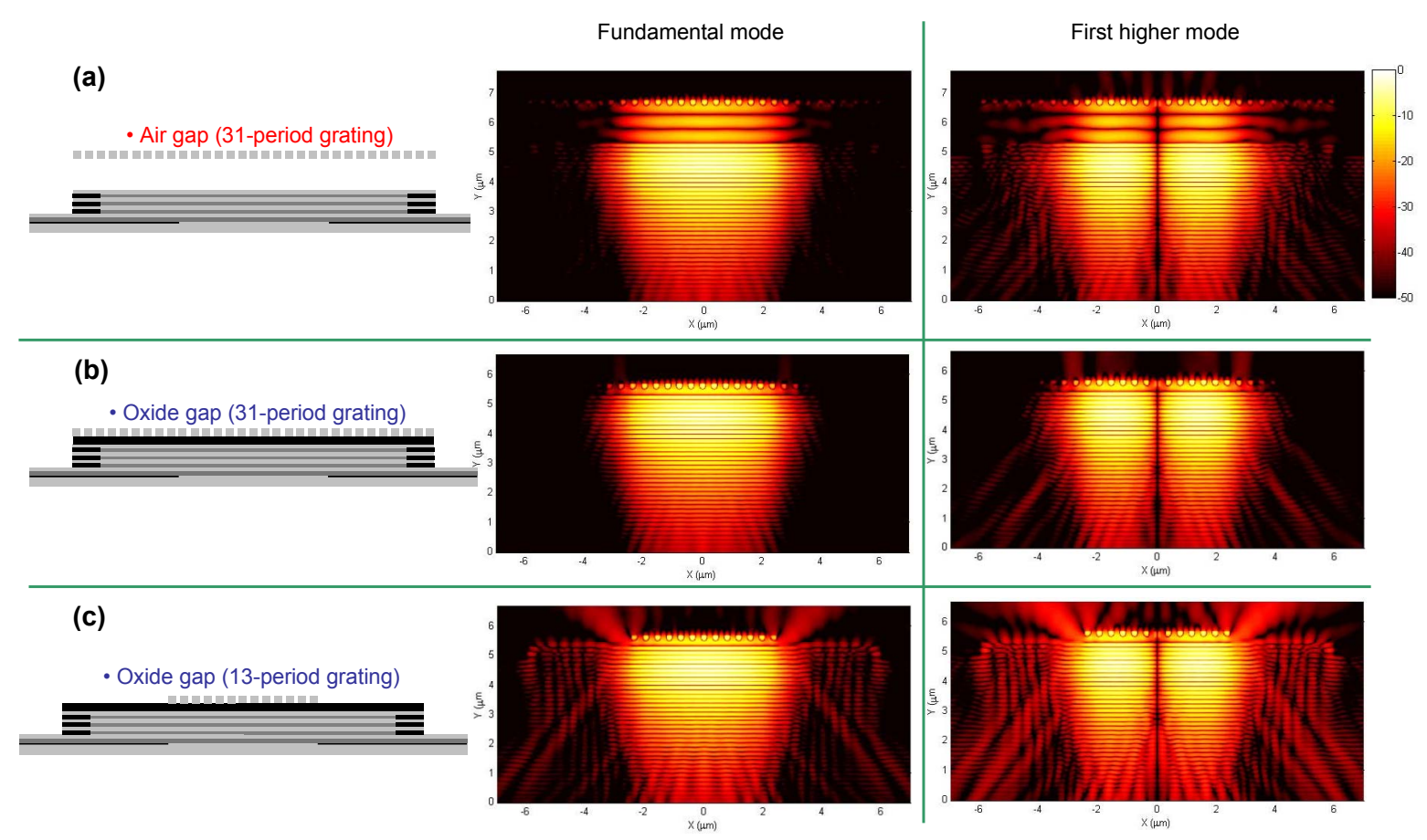

Figure 3. Mode profiles of fundamental and first higher order modes are plotted in $d B$-scale.

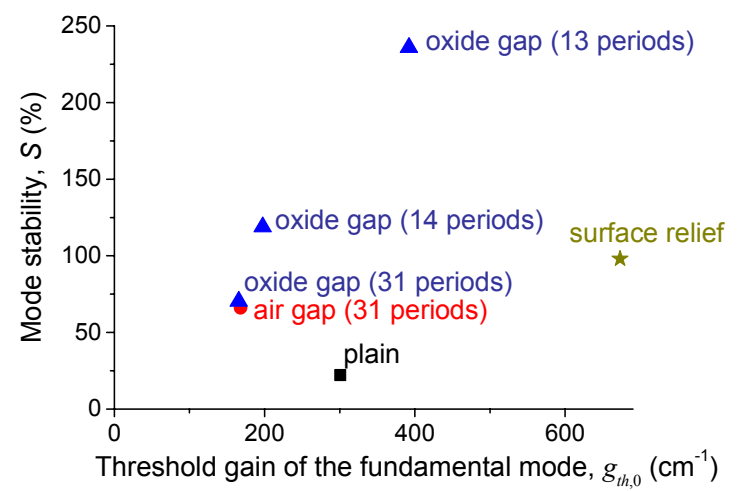

Figure 4. Mode stability vs. threshold gain.

\section{CONCLUSIONS}

Various HCG designs have been investigated, showing that TM-HCG is advantageous for thinner air or oxide gap. It is shown that a new VCSEL structure with a thin oxide gap exhibits performance characteristics similar to that of air gap based structures. An optimized grating mirror structure allows realizing very strong single-mode properties.

\section{ACKNOWLEDGEMENTS}

This work has been supported by EU IST-FP6 through the MOSEL project.

\section{REFERENCES}

[1] S. Boutami, et al.: Highly selective and compact tunable MOEMS photonic crystal Fabry-Perot filter, Optics Express, vol. 14, pp. 3129-3137, Jan. 2006.

[2] H. T. Hattori, et al.: Analysis of hybrid photonic crystal vertical cavity surface emitting lasers, Optics Express, vol. 11, pp. 1799-1808, Jul. 2003.

[3] E. Bisaillon, et al.: High reflective air-bridge subwavelength grating reflector and Fabry-Perot cavity in AlGaAs/GaAs, Optics Express, vol. 14, pp. .2573-2582, Apr. 2006.

[4] M. C. Y. Huang, et al., A surface-emitting laser incorporating a high-index-contrast subwavelength grating, Nature Photon., vol. 1, pp. 119-122, Feb. 2007.

[5] I.-S. Chung, et al.: Subwavelength grating-mirror VCSEL with a thin oxide gap, IEEE Photonic. Technol. Lett., vol. 20, pp. 105-107, Jan. 2008. 